Review

\title{
Recent Progress for the Techniques of MRI-Guided Breast Interventions and their applications on Surgical Strategy
}

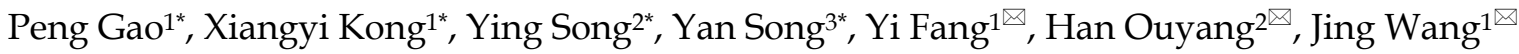

1. Department of Breast Surgical Oncology, National Cancer Center/National Clinical Research Center for Cancer/Cancer Hospital, Chinese Academy of Medical Sciences and Peking Union Medical College, Beijing, 100021, China.

2. Department of Diagnostic Radiology, National Cancer Center/National Clinical Research Center for Cancer/Cancer Hospital, Chinese Academy of Medical Sciences and Peking Union Medical College, Beijing, 100021, China.

3. Department of Pathology, National Cancer Center/National Clinical Research Center for Cancer/Cancer Hospital, Chinese Academy of Medical Sciences and Peking Union Medical College, Beijing, 100021, China.

*These authors contribute equally to this article.

$\square$ Corresponding authors: Jing Wang. Department of Breast Surgical Oncology, National Cancer Center/National Clinical Research Center for Cancer/Cancer Hospital, Chinese Academy of Medical Sciences and Peking Union Medical College, Beijing, 100021, China (E-mail: wangjing@cicams.ac.com); Yi Fang. Department of Breast Surgical Oncology, National Cancer Center/National Clinical Research Center for Cancer/Cancer Hospital, Chinese Academy of Medical Sciences and Peking Union Medical College, Beijing, 100021, China (E-mail: fangyi@cicams.ac.com); Han Ouyang. Department of Diagnostic Radiology, National Cancer Center/National Clinical Research Center for Cancer/Cancer Hospital, Chinese Academy of Medical Sciences and Peking Union Medical College, Beijing, 100021, China (E-mail: houybj@126.com).

(c) The author(s). This is an open access article distributed under the terms of the Creative Commons Attribution License (https://creativecommons.org/licenses/by/4.0/). See http://ivyspring.com/terms for full terms and conditions.

Received: 2020.03.25; Accepted: 2020.05.09; Published: 2020.05.20

\begin{abstract}
With a high sensitivity of breast lesions, MRI can detect suspicious lesions which are occult in traditional breast examination equipment. However, the lower and variable specificity of MRI makes the MRI-guided intervention, including biopsies and localizations, necessary before surgery, especially for patients who need the treatment of breast-conserving surgery (BCS). MRI techniques and patient preparation should be first carefully considered before the intervention to avoid lengthening the procedure time and compromising targeting accuracy. Doctors and radiologists need to reconfirm the target of the lesion and be very familiar with the process approach and equipment techniques involving the computer-aided diagnosis (CAD) tools and the biopsy system and follow a correct way. The basic steps of MRI-guided biopsy and localization are nearly the same regardless of the vendor or platform, and this article systematically introduces detailed methods and techniques of MRI-guided intervention. The two interventions both face different challenging situations during procedures with solutions given in the article. Post-operative statistics show that the complications of MRI-guided intervention are infrequent and mild, and MRI-guided biopsy provides the pathological information for the subsequent surgical decisions and MRI-guided localization fully prepared for follow-up surgical biopsy. New techniques for MRI-guided intervention are also elaborated in the article, which leads to future development. In a word, MRI-guided intervention is a safe, accurate, and effective technique with a low complication rate and successful MRI-guided intervention is truly teamwork with efforts from patients to surgeons, radiologists, MRI technologists, and nurses.
\end{abstract}

Key words: Breast cancer, MRI-guided, Breast surgery, Breast biopsy, Preoperative needle localization

\section{Introduction}

Breast cancer ranks first among all female tumors, and it tends to be diagnosed at a younger age than other common cancers, with a great threat to women's health and safety [1]. The early diagnosis and surgical treatment of breast cancer are of vital importance, especially for young women with the treatment of breast-conserving surgery (BCS, also known as partial mastectomy or lumpectomy). Magnetic resonance imaging (MRI), as an integral tool that is used in combination with mammography and 
ultrasound, has a sensitivity ranging from $86 \%$ to $100 \%$ in the detection of breast lesions [2-5]. MRI remains the most powerful tool for detecting clinically suspected lesions which are mammographically and sonographically occult. However, the specificity of MRI is between $37 \%$ and $97 \%$ [6-9]. The lower and variable specificity is primarily due to considerable overlap in the appearance of benign and malignant lesions. Thus, breast MRI is often performed as a preoperative examination for suspected lesions that can't be seen with mammography and ultrasound to assess the disease extent. Moreover, MRI-guided localization and biopsy of suspicious lesions are often needed before the surgery, particularly for the young women who deserve to receive BCS. Besides, MRIguided intervention is a valid technique for the diagnosis of suspicious non-palpable lesions (NPLs) and small masses, which is easy to cause misdiagnosis and delay the treatment of the diseases. These MRIguided interventions, which are safe, accurate, and effective with a low complication rate that includes infection, hematoma information, and pneumothorax, have transformed the practice of breast imaging [10-14].

Since the 1990s, MRI has been used for breast cancer screening and staging. In 2010, the American College of Radiology (ACR) required facilities to have the equipment to have MRI-guided interventions or have an agreement with the referral center to do so. Successful MRI images analysis for breast tumors is a crucial component for breast surgery $[2,15,16]$. This article reviews the indications and applications of MRI in breast tumors; Reviews the fundamental approaches and necessary steps for performing MRIguided biopsy and localization in breast tumors, discuss technical challenges in difficult situations with strategies and explores new techniques used in MRIguided interventions.

\section{Brief introductions of MRI-guided detection and therapeutic methods in human breast}

Apart from mammography and ultrasound, women with the most significant risks for developing breast cancer are recommended to have an MRI examination. MRI can give significant additional advice that single or multicentric diseases may not be visible or be detected with traditional breast lesion screening equipment, with a precise determination of the extent of the disease (Figure 1). A retrospective review showed that routine pretreatment MRI was not associated with delay in care, with initial surgery occurring in approximately the same interval after diagnosis, and margin-negative surgery actually being accomplished more quickly in patients receiving MRI [17].

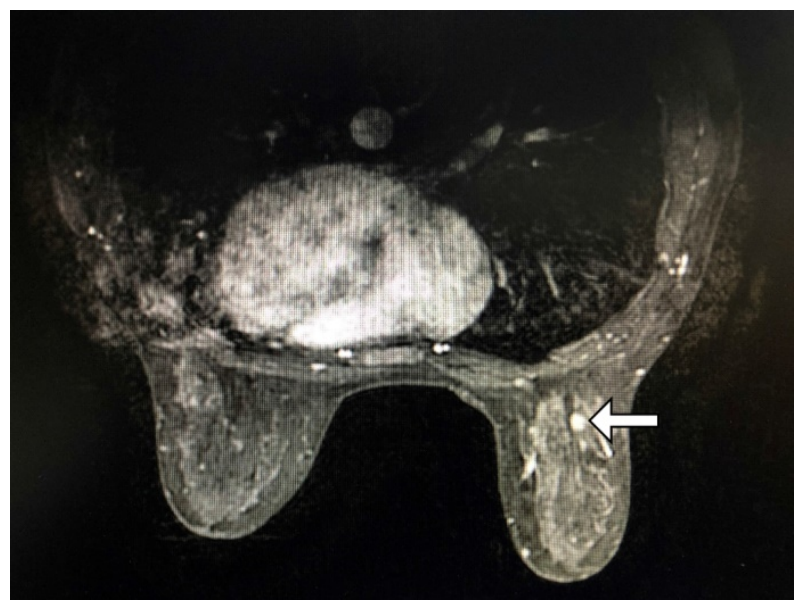

Figure 1. Examples of MRI-detected single right breast lesion of a 47-year-old woman (white horizontal arrow), which is invisible on the corresponding ultrasound and mammography, and the radiologist's report shows BI-RADS 4. This lesion can't be seen on ultrasound after re-identification and then MRI-guided localization is performed with a wire. Pathology after surgery revealed medium nuclear grade ductal carcinoma in-situ.

What's more, breast MRI has the advantage to detect lesions for high-risk patients. Breast MRI is considered in patients with a lifetime risk of $15-20 \%$, including patients with BRCA1 or BRCA2 mutation, a first-degree relative with a BRCA1 or BRCA2 mutation but who are themselves untested. A personal history of breast or ovarian cancer, and women with a history of high-risk breast lesions such as lobular neoplasia or atypical ductal hyperplasia; patients with a new diagnosis of breast cancer, a known tumor undergoing neoadjuvant chemotherapy, and metastatic lymphadenopathy with an unknown primary cancer are also recommended [18-20].

\section{Techniques of MRI in Breast}

It is better to perform the breast MRI between Day 3 and Day 14 in a typical 28-day menstrual cycle to limit the breast background parenchymal enhancement (BPE) which can render dynamic breast MRI examinations challenging to interpret, due to false-positive findings [21,22]. The diagnostic breast MRI is typically performed at either $1.5 \mathrm{~T}$ or $3 \mathrm{~T}$ magnetic field strength, and it is generally performed with patients in the prone position using a dedicated breast array coil which can reduce the distortion from respiratory variation when obtaining sequences. An endovenous injection of a paramagnetic contrast agent (such as gadolinium) is usually needed for both morphologic and kinetic features study [23]. Although the exact complete diagnostic protocol for breast MRI varies from different institutions, it is often composed of the following sequences: T2 fatsuppressed, pre-contrast T1 no-fat-suppressed, pre- 
contrast T1 fat-suppressed, and then several postcontrast $\mathrm{T} 1$ fat-suppressed images. These examinations take about 40 minutes to complete and vary with the number of sequences acquired [20]. Advanced imaging techniques such as diffusionweighted imaging (DWI) and MR spectroscopy provide complementary information.

Findings are read independently by different breast radiologists with years of experience in breast imaging. They assess the lesions' type (mass, nonmass enhancement, or focus), size, position, shape (circumscribed or irregular), marginal characteristics, and enhancement kinetics. Other characteristics also have to be recorded if not previously logged in the original report. If disagreements are among radiologists with the original report, they need to review the study to reach consensus. All MRI imaging studies are read according to the Breast Imaging Reporting and Data System (BI-RADS) and MRI directional ultrasound is recommended for suspected enhanced lesions (BI-RADS 4 or 5). Final patient treatment is based on an additional conjoint reading of results of all imaging studies [24-26].

\section{Challenging Clinical Situations}

The prone position can help to move the breasts away from the chest wall and minimize respiratory and cardiac motion effects [27]. However, the prone position acquires the breasts to be pendant into the coils or fixation plates from immobilization, which is different from the position and situation in the operating room (OR), especially when the surgeon is going to perform BCS. The supine position of patients remains the breast in its native configuration, so the difference between two positions may influence the surgeon when he uses the breast MRI for image guidance of BCS, and the surgeon has to mentally correct for the differences in orientation. In order to overcome this limitation, breast MRI in the supine position has been proposed [14]. In one study, patients lie flat on the MR bed with a fixture holding the imaging coil above breasts [28]. In this way, the position is more similar to the OR without any constrain or compression, which falls into its native configuration. Besides, the arms of patients have to be parallel to the body while the arms are placed nearly perpendicular to body axes during surgery, which is also a question because the positional change of the arm might cause a non-rigid-body deformation of the adjacent breast [11,29].

\section{Main technical issues of MRI-guided detection and therapeutic methods in human breast}

increasingly over the past decades for its high sensitivity and ability to detect clinically mammographically, and sonographically occult breast cancers. However, due to the lower and variable specificity of MRI, normal breast tissue and other benign lesions can also be enhanced in MRI. So suspicious lesions screened in MRI should be made to re-identify the lesion on mammography or ultrasound to determine whether the lesion can be found again. If the lesion can be re-identified, radiologists firstly choose to perform imaging-guided localization or biopsy with ultrasound and mammography rather than MRI due to the long duration and higher cost, and if a lesion is not clearly identifiable on US or mammography, MRI is used for guidance. Thus, the need for MRI-guided procedures is relatively limited in many institutions [30-33].

But the presumed correlate depicted on the ultrasound image does not truly represent the lesion noted on MRI [34]. Amie Y. Lee et al. [35] found that the presumed sonographic correlate was not the true correlate in $26 \%$ of 38 cases and that subsequent MRIguided biopsy of the actual lesion detected on MRI revealed one malignancy in discordant cases. These related researches prove the necessity of the MRIguided intervention.

\section{Preparations before MRI-guided Intervention}

Before the MRI-guided biopsy or localization, surgery and procedure; radiologists should review clinical information, standard breast imaging, and contrast-enhanced bilateral MRI to establish the quadrant, clock position, depth, distance from nipple, breast density, and surrounding breast tissue landmarks. Other factors which may be assessed include determination of best approach, communication with technologists, optimization of patient positioning, preprocedural checklist confirmation, and accurate targeting of the lesion, which are also critical for maximizing the chances of a safe and successful intervention $[2,36]$.

To improve the efficiency, the intervention equipment, such as the configuration of the coil, and patient body habitus which can influence the biopsy approach, should be prepared before a patient enters the MR imaging suite (Figure 2). Besides, the placement of grids which determine the access of the intervention should be carefully prepared. Gentle breast fixation is used to avoid the lack of enhancement of a lesion, and because of the compression of the breast, it is necessary to carefully evaluate the parenchyma surrounding the enhancing target for reliable anatomical landmarks [18].

The breast MRI has been used clinically 

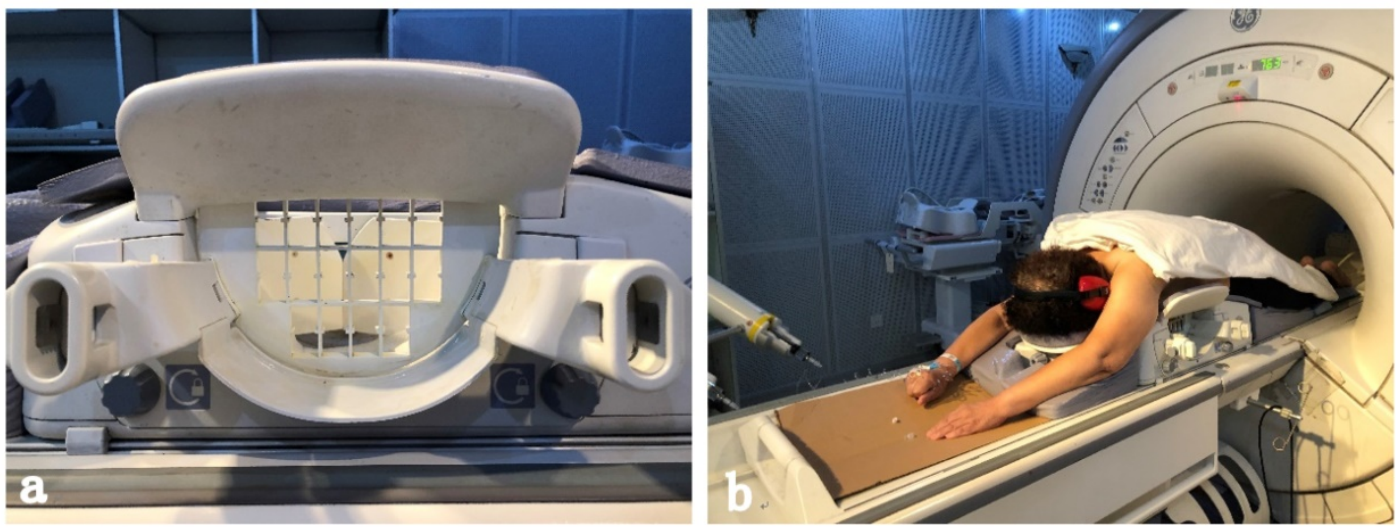

Figure 2. Preparations are significant for successful MRI- guided breast intervention. The coil (a)which is designed for MRI-guided intervention. A Patient is positioned prone in the coil (b) for the examination of MRI, with a mild compression for the breast. A headphone connected to the machine is used to reduce the noise of the MRI room. The arms of the patient are positioned above the head to avoid wrap artifacts and some newer coil techniques allow the arms to be positioned at the sides.

For patients, receiving MRI-guided interventions are usually regarded as an intense, even scary experience, so before written informed consent, it's vitally important for surgeons and radiologists to communicate with their patients to discuss the reasons for the requested intervention, and the possible events that might occur during the procedure. Goals, benefits, the expected time the procedure might take and potential risks, and the possibility of cancellation should be explained before the operation. A study reported that listening to guided meditation lowered biopsy pain during the biopsy and that meditation and music reduced patient anxiety and fatigue [37]. The patient collaboration is the key to the success of the procedure because the patient should remain still during the procedure, so the comfort of the patient during this period is essential to avoid lengthening the procedure time and compromising targeting accuracy. The patient should also be warned about the concept of non-visualization and the possibility, depending on lesion location within the breast, that there will be no safe access for sampling or localization [16,38]. Of course, radiologists and technologists mush check patients who have any contraindications to MRI and/or gadolinium-based contrast administration when performing any contrast-enhanced breast MRI, during the previous breast MRI examination or the current MRI-guided intervention [39].

Successful MRI-guided intervention is truly a teamwork effort from patients to the surgeons, radiologists, MRI technologists, and nurses.

\section{Lesion Identification and Targeting}

Radiologists should confirm triplane localizer images before the day of the intervention. Precontrast and postcontrast T1-weighted fat-saturated gradient echo sequences are performed. A fiducial marker (visible on T1- weighted sequences) is usually within the grid to facilitate lesion localization. The pre-contrast sequence should be reviewed to verify the approximate location of the lesion and the quality of fat saturation, the grid and the fiducial marker. Although some coils have standard locations where the fiducial is placed, put the fiducial in a grid square that is relatively close to the approximate location of the lesion but unlikely to be the exact entry location is recommended [40]. And it is important that the imaging protocol should minimize image acquisition time while maintaining lesion visualization, because there is a short period following the administration of the intravenous contrast during which the area of interest can be visualized [41,42].

The coordinates of the chosen biopsy site can be subsequently calculated from computer software [eg, commercially available computer-aided diagnosis (CAD) tools] or manual localization method, and both methods of localization are usually used together. CAD system can assist radiologists in the analysis of breast MRI to reduce the interpretation time of analyzing breast MRI data. Albert Gubern-Mérida et al. [43] propose a novel CAD system to detect breast lesions in dynamic contrast-enhanced MRI automatically. As for the manual localization method, landmarks including: vessels, biopsy clips, and other foci of background parenchymal enhancement can be used to help localize the target [12].

To target the lesion, the grid-guidance system and fiducial marker are adopted to determine the lesion coordinates (the $\mathrm{x}, \mathrm{y}$ and $\mathrm{z}$ axes). Also, the CAD software can be used to calculate the skin entry site, select the correct needle insertion depth, and determine the optimal needle type, which can short the procedural time (Figure 3). Radiologists can obtain depth ( $\mathrm{z}$ axes) of the lesion by counting the number of images from the skin surface where the grid is visualized to the targeted lesion and multiplying that number by the slice thickness. 
Remember that the skin surface is designated as a slice where the grid indentations on the skin are best visualized. Besides, a postcontrast axial, fat-saturated T1 sequence can also be used to measure lesion depth from the overlying skin. Alternatively, built-in scanner software can be used to records the depth coordinates for the lesion and the fiducial $[2,18]$.

\section{Aseptic Technique before the Intervention}

Before the intervention, technologists should clean and anesthetize the entry site using the sterile technique [44]. Pain associated with local anesthetic administration may be improved by slow injection and buffering with sodium bicarbonate $(8.4 \%)$, oral pain medication administered to the patient before the procedure, or i.v. sedation [23]. For superficial anesthesia, lidocaine buffered in sodium bicarbonate may be used to reduce the initial stinging sensation of the lidocaine injection. And alternative drugs have to be used if the patient is allergic to lidocaine [45].

\section{Specific applications of MRI-guided detection and therapeutic methods in human breast}

\section{MRI-guided Biopsy}

\section{Biopsy system}

When performing the MRI-guided biopsy, it is significant for radiologists and MRI technologists to be very familiar with the components of the coaxial biopsy system and follow a correct way.

Vacuum-assisted large-gauge systems are recommended for biopsy, due to reasons of the challenges in precise targeting, the difficulties of MRIguided biopsy and confirming lesion retrieval. It is crucial to select suitable commercial devices which are available from 8-12 G sizes, in order to optimize tissue sampling when targeting enhancing lesions that are in challenging positions [46].

The biopsy system generally contains: (1) an outer plastic introducer trocar/sheath with numbers indicating insertion depth and with an adjustable depth stopper, (2) a cube-shaped plastic tunnel needle guide that fits into the biopsy grid, (3) a cutting introducer stylet that helps to advance the plastic introducer trocar/sheath to the appropriate depth, and (4) a plastic obturator that is used to indicate the center of the biopsy chamber of the vacuum-assisted breast biopsy device on prebiopsy images (Figure 4) $[18,47]$.

Adequacy of sampling is significant during the biopsy. It is typically performed with 12 samples (usually 1 sample per o' clock position), and additional samples may be obtained if the targeting is offset $[2,48]$. The biopsy device usually allows manual rotation of the biopsy needle to sample the lesion preferentially. During the procedure, operators must always remember that the clockface is relative to them and it may be easier to direct sampling towards the body part (e.g. head, nipple, feet, or chest wall) or landmark (e.g. floor or ceiling). When completing the sample, lavage and aspiration of the biopsy cavity are performed to minimize the risk of post-biopsy hematoma and reduce imaging artifacts at the biopsy site. Tissue samples are placed in $4 \%$ formaldehyde and sent for histopathology $[6,49]$.

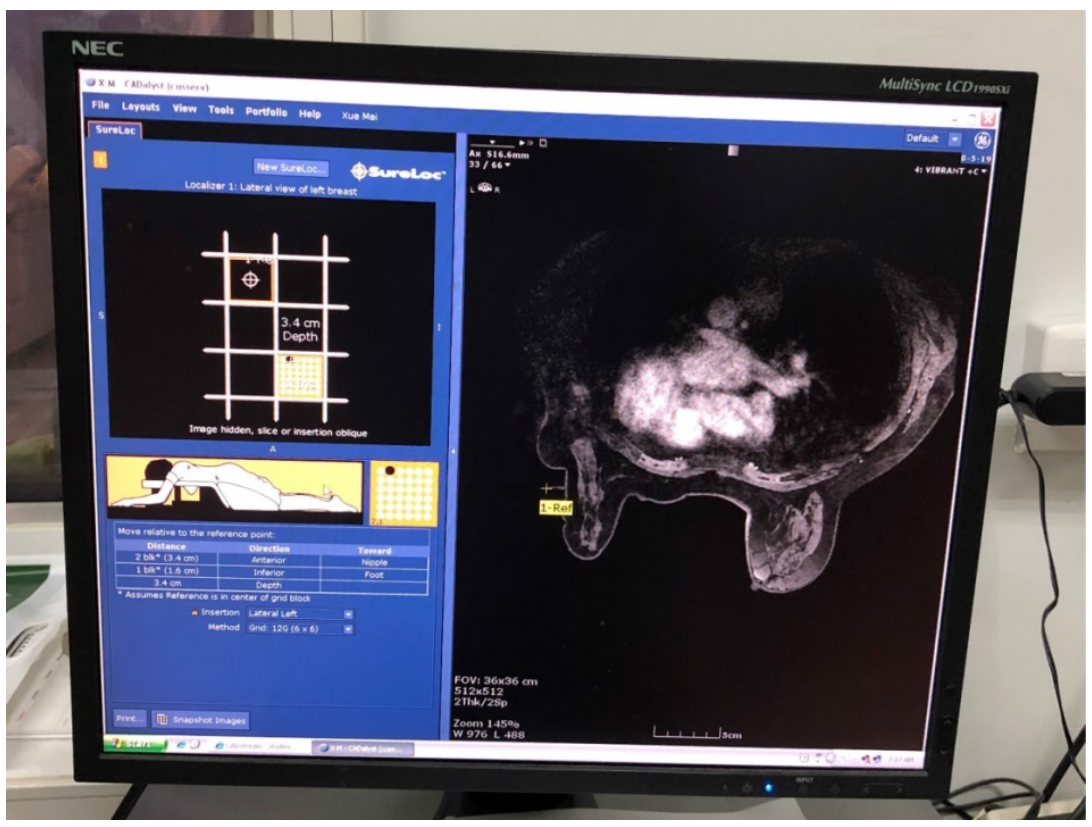

Figure 3. Computer-aided targeting in a 47-year-old woman with right breast lesion which is identified in the computer-aided detection (CAD) system. The CAD system provides a graphic depicting the optimal entry point for the needle (yellow crosshairs), and it also calculates coordinates of the lesion towards the nipple and foot. Especially, the lesion depth is important for MRI-guided intervention. 


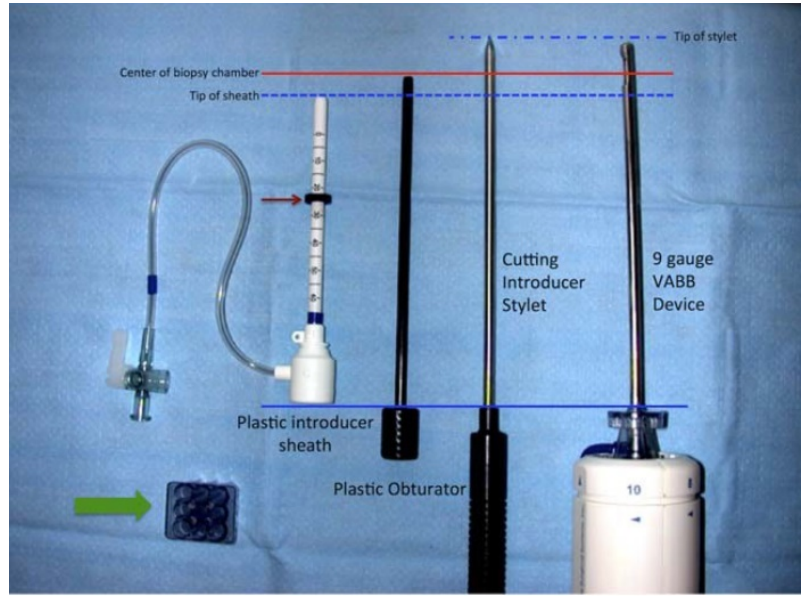

Figure 4. Components of an MRI-compatible coaxial biopsy system. The plastic introducer sheath is placed into the tunneled needle guide (wide green arrow) and advanced to the appropriate depth within the breast using a cutting introducer stylet. A rubber stopper (narrow red arrow) is used to set the appropriate depth. In this system, the plastic obturator is used to indicate the center of the biopsy chamber of the vacuum-assisted breast biopsy (VABB) device (solid red line) on prebiopsy images. Reproduced with permission. Modified and reprinted from McGrath AL, Price ER, Eby PR, Rahbar H. MRI-guided breast interventions. J Magn Reson Imaging. 2017. 46(3): 631-645. Copyright (C) 2017 International Society for Magnetic Resonance in Medicine.

Lastly, MRI-compatible metallic clip, visible on MR, mammography and ultrasound, is deployed at the biopsy site upon removal of the biopsy device. Metallic clips are usually used to mark the lesion in case there is a need for additional needle biopsy or excision following the pathology report. For cancer patients who choose to have breast-conserving surgery, MRI-guided clip placement of a broad area of known disease or adjacent satellites before neoadjuvant therapy facilitates preoperative localization. A rapidly developing hematoma may interfere with further sampling, so the metallic clip should be placed, and the hematoma should be managed immediately. A post-biopsy T1-weighted image is then obtained to ensure the lesion is accurately targeted and it also allows the radiologist to confirm the placement of the clip. Whether to confirm the position of the clip with an MRI sequence has no consensus. However, a two-view mammogram is recommended which can help to confirm clip mark placement due to the postsurgical blood products and air can also obscure the susceptibility artifact. Because the compression of the breast may make a distortion, the location of the marker is not necessarily a reliable assessment of the sampling accuracy $[2,16]$. Any clip displacement should be estimated and documented under the comparison of mammography and MRI. Li et al. [50] observed that the distance between the metallic clip and the lesion was not a reliable indicator of biopsy accuracy (Figure 5).

\section{Challenging Clinical Situations}

Some clinical situations may be somewhat challenging for technologists to have MRI guided biopsy. As for superficial lesions, a "half window" of sampling aperture is usually used in many systems to minimize skin damage and a blunt-tipped needle can be used to avoid skin damage [16]. If the grid obscures the optimal entry site, causing the needle entry to be offset to the underlying target, the directional sampling method may also be needed [2]. In some institutions, the freehand method is used for MRI-guided breast biopsy, which is different from other facilities that use a grid and contrast enhancement prior to needle placement. This method does not need specialized grid devices, and it allows for lesion localization throughout the breast, including lesions near the chest wall, in the axillary region and markedly posterior lesions [42,47]. Lee JM et al. [51] reported an $8 \%$ to $13 \%$ cancellation rate of the procedure. Non-visualization of the suspicious finding may be due to change in tissue enhancement as the patient is in a different phase of her period or may be related to compression of breast tissue with decreased inflow of contrast material [52].

When multiple lesions are required to be biopsied, more than one technologist or radiologist may be helpful, and the biopsy supplies or equipment should be set up in advance. The biopsy devices should be available to be quickly set up once the targets are visualized to take advantage of the enhancement time and to prevent errors caused by patient movement.

Biopsy for women with breast implants needs to have informed consent for the risk of implant rupture. Dedicated technologists may provide invaluable help in displacing the implant to minimize the risk of implant rupture and ensure the adequacy of sampling $[2,16]$.

\section{MRI-guided Localization}

\section{Techniques of MRI-guided Localization}

In addition to MRI-guided biopsy, radiologists and breast surgeons should be familiar with MRIguided localization. MRI-guided localization allows guidance of surgeons to the lesion to obtain both a complete removal (clear margins) and a good cosmetic result [53]. Preoperative localization is often performed when MRI-guided biopsy is not feasible due to technical factors or patient's preference, such as the target lesion that is not accessible with MRIguided biopsy $[4,54]$. This approach is often needed by breast surgeons to help them perform breastconserving surgery when the lesions are only visible with MRI, and to guide surgical re-excision of residual disease in cases of positive margins. Besides, MRIguided localization is also used to demarcate lesions adjacent to benign enhancing structures, such as a scar 
or breast implants [24]. And when neoadjuvant therapy is under consideration in order to mark the lesion site for re-evaluation or treatment planning, MRI-guided localization can also be performed [55].

When the coordinates of the target have been identified through computer software which is in the previous description, the skin entry site and optimal needle trajectory would be selected, and the needle guide and the needle are introduced through the grid hole overlying the skin [56]. In the method of freehand technique, the needle trajectory should base on the desired surgical approach, and a full range of angled paths within the axial plane can be available as far as possible. The location of consequential and special structures should be avoided, such as implants. The skin entry site is marked with a pen after lesion identification [54,57].

After sterilization and anesthetization, a needle guide is used for accurate wire placement, and an MRI-compatible hook needle is inserted into the marked skin entry site and directed along with the planned needle approach to the appropriate location with the help of the grid-localization system. If a needle guide was used, an additional depth (typically $2 \mathrm{~cm}$ ) should then be added to the calculated depth [2]. The necessary steps of MRI-guided localization are almost the same as the process of MRI-guided biopsy, and MRI-guided localization allows for conservative surgical excision of a limited amount of tissue, yielding together an effective treatment and good aesthetic results [18,58-60]. During the procedure of MRI-guided localization, dynamic real-time MR imaging can be performed to help visualize the placement of the needle and the release of the guidewire. Moreover, the radiologist can see and adjust the procedure of localization through a monitor that displays the MR images in the imaging room. The position of the needle can be immediately corrected if a shift of the breast tissue or a deviation of the needle is visible under the control of real-time MR imaging. The $1-\mathrm{cm}$ marks on the shaft of the MR-compatible needle can be used to adjust the needle's position [61]. Finally, when the position and the calculated depth are deemed satisfactory, the insertion of the needle is stopped, and the guide hook wire is released.

The hook of the wire should be located beyond the target, which is analogous to that of mammographic-guided wire localizations. But due to that the compression of the breast is in the same direction as the wire placement approach in the MRI coil, MRI-guided wire localizations are more prone to postprocedural wire migration than mammography [18]. Postprocedure two-view mammography should then be performed to confirm the position of the wire prior to surgery and to help the breast surgeon in determining the wire position (Figure 6) [62].

\section{Challenging Clinical Situations}

For multiple lesions and bilateral lesions, localization is usually performed of the more suspicious lesion or the smaller lesion, followed by localization of the remaining lesion. The localization of superficial target lesions is often performed using a particular marker, such as vitamin E, over the skin because there is not enough tissue depth to adequately secure a localization wire in place [24]. For lesion close to the nipple, where the nipple may be difficult to stabilize, the needle may be placed adjacent to the lesion instead of through the lesion [56].

Nonvisualization may occur when radiologists perform the MRI-guided intervention at the rate of $12-13 \%[63,64]$. The majority of these lesions likely represent fluctuating physiologic $\mathrm{BPE}$, but the absence of enhancement does not exclude malignancy. ACR Practice Parameters recommend "short-term follow-up," and 3-6 months MRI follow-up is often suggested [18]. MRI-guided intervention should be performed if the lesion is reappeared on follow-up, especially if the lesion is enlarged or shows malignant signs.

\section{The combination of MRI-guided methods and other technologies in human breast}

\section{Considerations after the Operation}

Once the procedure has been finished, the radiologist initiated manual hemostasis within the MRI suite. The puncture location of the breast should be sterilized again, and manual compression, usually for $5 \mathrm{~min}$, as well as cooling, is applied followed by a compressive dressing. Then the patient is moved out of the magnet and kept warm with clothes. It is generally considered that half an hour of monitoring after the surgery is usually sufficient, and the radiologist should provide the patient with postprocedural care instructions [41,65]. Besides, efforts also should be made to decrease patients' distress and anxiety, which is important general aspects for patients and the quality of the patient-doctor relationship [66]. 

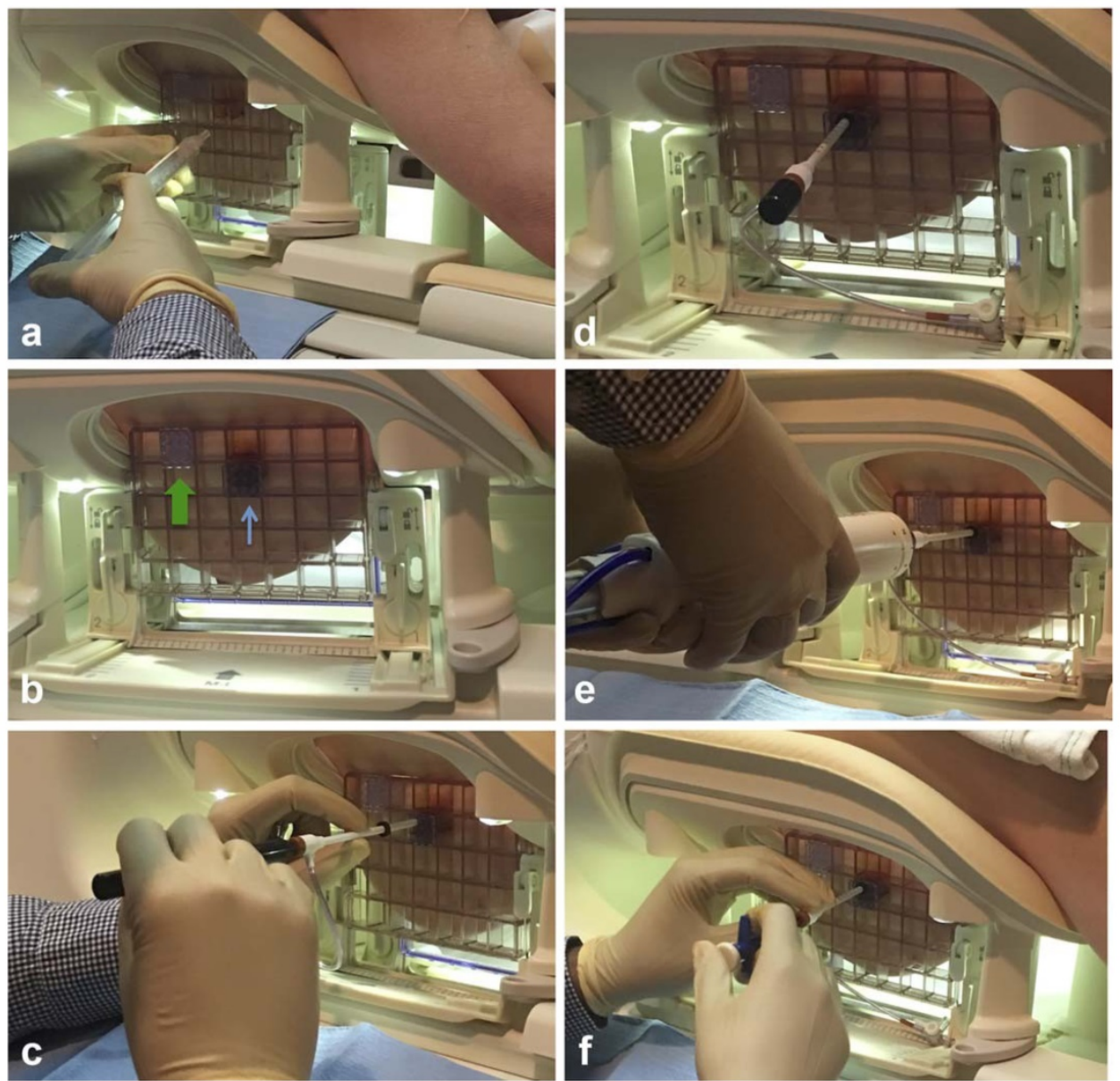

Figure 5. Example of typical steps involved when performing an MRI-guided breast biopsy. After determining the appropriate grid space for the skin entry site, the skin is cleansed and anesthetized (A), and local anesthetic is administered into the deeper breast tissues. The tunneled needle guide is then placed in the grid space where the biopsy (thin blue arrow) noting location relative to the fiducial (wide green arrow) (B). Using a "twist and push" motion, the plastic introducer sheath is placed into the breast through the appropriate hole in the tunneled needle guide with the cutting introducer stylet inside the sheath until the sheath reaches the appropriate depth as indicated by the rubber stopper (C). The cutting introducer stylet is subsequently replaced with a plastic obturator, and the patient is placed back in the bore of the MRI scanner so that a confirmatory $\mathrm{Tl}$-weighted sequence can be performed to confirm the appropriate location (D). The table is then brought out of the bore and the plastic obturator is replaced with the vacuum-assisted breast biopsy (VABB) device and multiple cores are obtained (E). After the sampling is complete, the VABB device is removed and a biopsy marker clip is deployed through the sheath (F). Modified and reprinted from McGrath AL, Price ER, Eby PR, Rahbar H. MRI-guided breast interventions. J Magn Reson Imaging. 2017. 46(3): 631-645. Copyright (C) 2017 International Society for Magnetic Resonance in Medicine.

(A)

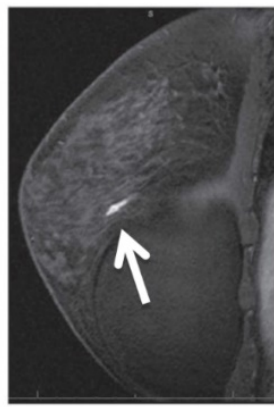

(B)

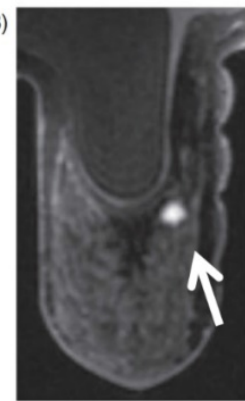

(C)

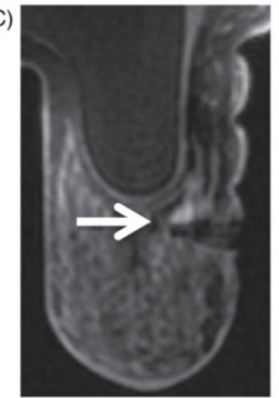

(D)

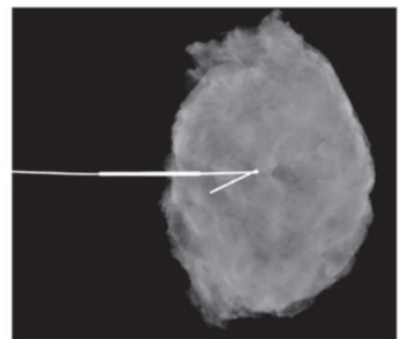

Figure 6. A 43-year-old female with a strong family history of breast cancer. A and B, Sagittal and axial T1-weighted postcontrast images from screening MRI with an enhancing mass anterior to the left implant (arrows). There was no sonographic correlation. C, Axial T1-weighted postcontrast MRI shows successful MRI-NL (arrow). D, Specimen radiograph shows the wire in place after excisional biopsy. Pathology showed pseudoangiomatous stromal hyperplasia and fibroadenomatoid changes. Reproduced with permission. Modified and reprinted from Raj SD, Agrons MM, Woodtichartpreecha P, et al. MRI-guided needle localization: Indications, tips, tricks, and review of the literature. Breast J. 2019. 25(3): 479-483. Copyright @ 2019 Wiley Periodicals, Inc.

\section{New Techniques for Lesion Localization and MRI-Guided Breast Interventions}

Currently, techniques available for MRI-guided intervention are developing quickly, and the market for alternative methods is increasing. With the increase of patients who is eligible for breast MRI, faster, cheaper and accurate MRI techniques will also be needed.

In order to make the MRI scan faster, many are 
investigating "ultrafast" techniques that complete postcontrast imaging within 30 seconds of contrast injection $[67,68]$. Breast MRI offers the potential for the measurement of fibroglandular tissue volume to assess breast density, and Gokhan E et al. [69] developed a fast, computerized algorithm for volumetric breast segmentation, suitable for multicenter data, employing 3D bias-corrected fuzzy cmeans clustering and morphological operations to make the assessment more accurate. Moreover, statistical analysis shows that the segmentation algorithm performs well when compared with manually corrected segmentation in a UK multicenter study of MRI screening with eighty-two women scanned.

Apart from contrast-based MRI protocols, non-contrast MRI techniques, such as diffusionweighted imaging (DWI), are developing. As a functional MRI technique, DWI has the benefit of not requiring intravenous gadolinium, takes less time and is of lower cost than a full protocol traditional contrast-enhanced MRI. Besides, lesion detection and characterization, and the evaluation of neoadjuvant treatment response of DWI are also promising applications [70-72]. Nicole B et al. [49] evaluated the feasibility of DWI for lesion targeting in MRI-guided biopsies, and concluded that the targeted lesion requires the characteristics of a mass-like lesion, substantial diffusion restriction, and a minimum size of approximately $1 \mathrm{~cm}$.

Samuel BP et al. [73] developed an MRcompatible needle intervention robotic system, which can approach the tumor from the side with a bendable needle. This new design solves the space limitations imposed by the MR gantry and breast coils. The position accuracy is increased with the system's image-guided feedback software.

With the preoperative supine MRI, Barth RJ Jr et al. [74] outline the tumor edges on consecutive images and create a bra-like plastic form (the Breast Cancer Locator [BCL]) that is manufactured for each patient using a $3 \mathrm{D}$ printer. The $\mathrm{BCL}$ can transmit both surface and intraparenchymal information to locate the tumor precisely in the OR and can help the surgeon to perform the breast-conserving surgery with the goal of removing $1 \mathrm{~cm}$ of normal-feeling tissue around the palpable tumor.

In addition to needle/wire localization aided guidance, in recent years, more and more new technologies are born that allow for localization up to several days before surgery, like carbon marking, radioactive technetium $-99 \mathrm{~m}(99 \mathrm{mTc})$ localization for lesion and sentinel lymph node, radioactive iodine-125 (125I) seeds localization, magnetic seed localization and SAVI SCOUT radar localization.
These new technologies can relieve the pressures of the number of same-day requests for diagnostic localization or biopsy of breast imaging centers, as well as increase patients' convenience and comfort. However, these newer techniques cannot be placed under MRI guidance, and to date only wire localizations can be performed under MRI guidance [75-81].

\section{Main advantages of MRI-guided detection and therapeutic methods in human breast}

MRI remains the most powerful tool for detecting clinically suspected lesions which are mammographically and sonographically occult. MRIguided biopsy or localization is essential in the management of suspicious breast lesions detected by screening or during the assessment of clinical abnormalities. It is a safe and cost-effective procedure allowing for an accurate diagnosis, pivotal for adequate decision-making, including, when indicated, treatment planning [82,83]. MRI has a sharp delineation of breast lesions, which is a right choice of the imaging modality to help the surgeon choose surgical approaches between BCS and mastectomy. Before performing BCS, the surgeon must use the MR images to confirm multicentric or single tumor of the breast, and review the size and location of the tumor combining with other equipment to ensure the success of the operation. Breast surgeon needs to minimize the amount of tissue excised and to ensure that clear surgical margins are as much as possible achieved before making the first incision[11, 84, 85]. Besides, MRI-guided biopsy is also used to prevent unnecessary surgery, associated morbidity and costs for equivocal findings on imaging with final non-malignant histopathology [86].

For MRI-guided intervention, complications are infrequent and mild [2]. Major complications during or after vacuum biopsy include infection, hematoma formation, pneumothorax and skin damage, with the complication rates ranging from 0 to $6 \%$ [65,87-90]. And these risks can be minimized with careful biopsy planning and selection of an appropriate biopsy device. The risk of mechanical displacement of malignant cells along the biopsy tract can very rarely occur and is referred to as neoplastic seeding. Brenner RJ et al. [91] reported that the risk of this event was to be 2 of 1644 biopsies, and Santiago L et al. [92] reported the incidence was to be 8 of 4010 biopsies.

For MRI-guided localizations, complication rates reported in the literature are $0 \sim 3.3 \%$ $[10,24,54,57,59,61,93]$. Complications consist of wire dislocations without relevance to the planned surgical procedures, bleeding out of the puncture channel, and 
hematoma, etc. Kathrin Barbara Krug et al. [10] performed 347 patients with MR-guided wire localization and reported that the complication rate is $3.3 \%$ and it was not influenced by patient age, clinical referrer, the indication of the wire localization, or histology of the operative specimen.

\section{Main disadvantages of MRI-guided detection and therapeutic methods in human breast}

The MRI-guided intervention is safe and accurate in specialized centers and relatively fast, but longer and more costly if compared to other imagingguided procedures.

For the biopsy, the average time spent in the MRI is from 20 to 90 minutes, and this is increased by 30 to $50 \%$ when two sites are biopsied [65,94-96]. Mitra Noroozian et al. [41] reported that the duration of the procedure depends on the number of images and checks in the tunnel, whether or not an assistant is present and proximity to the nipple, and it is independent of patient age, size of the breast and type or size of enhancement. For needle localizations, the entire operation lasts for about 40 minutes $[6,10,61,73,93,97]$. Krug KB et al. [10] warrant that it is beneficial to reduce the time of the interventional procedure to increase the patient's convenience during the intervention to avoid target lesion shadows due to contrast washout, to avoid inaccurate definition of target lesions due to motion artifacts, and to improve the ability of the MRI scanner.

\section{Perspectives and Conclusion}

Correlation between radiology and pathology is the final step after MRI-guided interventions, and it has unique challenges between benign and malignant lesions. For cases that have been assessed as possibly missed or discordant, a repeat biopsy or surgical excision is recommended. MRI-guided core biopsy malignancy rates varying between $16-40.91 \%$ have been reported [4,32,41,98-101]. For benign-concordant lesions, a diagnostic follow-up MRI after 6 months of MRI-guided interventions is recommended due to the risk of false-negative results [18,50]. And as for malignancy, doctors need to choose open surgical methods according to the condition of the patient.

MRI provides very high sensitivity for the detection of breast tumors which can't be seen with traditional breast examination equipment, based on anatomical and functional information, and it provides important guidance for the diagnosis and treatment of occult breast carcinomas. As for the subsequent treatment, preoperative MRI-guided intervention plays a vital role in the choice between
BCS and mastectomy. Whether benign or malignant concordant lesions, preoperative MRI-guided biopsy provides necessary information for doctors and patients with a strong willingness for breastconserving. Also, preoperative MRI-guided wire localization successfully and virtually ensures the performance of diagnostic excisions of suspicious breast lesions that can only be seen in MRI, and it also ensures the possibility of breast-sparing resections for the patients with single cancer of breasts.

Although MRI-guided intervention is more complicated, take more time and are more costly than other lesion image-aided localization technique. It offers the benefits high sensitivity rate for suspected lesions of MRI while minimizing false-positive findings and improving specificity by tissue sampling. What's more, it yields high technical and clinical success rates, low complication rates, and only minor forms of complication.

This work was supported by the Natural Science Foundation of China (No. 81872160), the Beijing Municipal Natural Science Foundation (Key Project) (No. 19G10077), the Beijing Municipal Natural Science Foundation (No. 7204293), the Special Research Fund for Central Universities, Peking Union Medical College (No. 3332019053), the Beijing Hope Run Special Fund of Cancer Foundation of China (No. LC2019B03), the Beijing Hope Run Special Fund of Cancer Foundation of China (No.LC2019L07), the CAMS Initiative for Innovative Medicine Foundation (No.2017-I2M-3-020).

\section{Competing Interests}

The authors have declared that no competing interest exists.

\section{References}

1. Miller KD, Siegel RL, Lin CC, Mariotto AB, Kramer JL, Rowland JH, et al. Cancer treatment and survivorship statistics, 2016. CA Cancer J Clin. 2016; 66: 271-89.

2. Santiago L, Candelaria RP, Huang ML. MR Imaging-Guided Breast Interventions: Indications, Key Principles, and Imaging-Pathology Correlation. Magn Reson Imaging Clin N Am. 2018; 26: 235-46.

3. Berg WA, Gutierrez L, NessAiver MS, Carter WB, Bhargavan M, Lewis RS, et al. Diagnostic accuracy of mammography, clinical examination, US, and MR imaging in preoperative assessment of breast cancer. Radiology. 2004; 233: 830-49.

4. Han BK, Schnall MD, Orel SG, Rosen M. Outcome of MRI-guided breast biopsy. AJR Am J Roentgenol. 2008; 191: 1798-804.

5. Mahoney MC. Initial clinical experience with a new MRI vacuum-assisted breast biopsy device. J Magn Reson Imaging. 2008; 28: 900-5.

6. Eliahou R, Sella T, Allweis T, Samet Y, Libson E, Sklair-Levy M. Magnetic resonance-guided interventional procedures of the breast: initial experience. Isr Med Assoc J. 2009; 11: 275-9.

7. Morris EA. Diagnostic breast MR imaging: current status and future directions. Magn Reson Imaging Clin N Am. 2010; 18: 57-74.

8. Kriege M, Brekelmans CT, Boetes C, Besnard PE, Zonderland HM, Obdeijn IM, et al. Efficacy of MRI and mammography for breast-cancer screening in women with a familial or genetic predisposition. N Engl J Med. 2004; 351: 427-37.

9. Saslow D, Boetes C, Burke W, Harms S, Leach MO, Lehman CD, et al American Cancer Society guidelines for breast screening with MRI as an adjunct to mammography. CA Cancer J Clin. 2007; 57: 75-89.

10. Krug KB, Ulhaas A, Hellmich M, Schwabe H, Kramer S, Malter W, et al. Impact of clinical and lesion characteristics on the results of MR-guided wire 
localizations of the breast using an open 1.0-T MRI system. Invest Radiol. 2013; 48: 445-51.

11. Ebrahimi M, Siegler P, Modhafar A, Holloway CM, Plewes DB, Martel AL. Using surface markers for MRI guided breast conserving surgery: a feasibility survey. Phys Med Biol. 2014; 59: 1589-605.

12. Chesebro AL, Chikarmane SA, Ritner JA, Birdwell RL, Giess CS. Troubleshooting to Overcome Technical Challenges in Image-guided Breast Biopsy. Radiographics. 2017; 37: 705-18.

13. Berg WA, Zhang Z, Lehrer D, Jong RA, Pisano ED, Barr RG, et al. Detection of breast cancer with addition of annual screening ultrasound or a single screening MRI to mammography in women with elevated breast cancer risk. JAMA. 2012; 307: 1394-404.

14. Alderliesten T, Loo C, Paape A, Muller S, Rutgers E, Peeters MJ, et al. On the feasibility of MRI-guided navigation to demarcate breast cancer for breast-conserving surgery. Med Phys. 2010; 37: 2617-26.

15. Patriciu A, Chen M, Iranpanah B, Sirouspour S. A tissue stabilization device for MRI-guided breast biopsy. Med Eng Phys. 2014; 36: 1197-204.

16. Chevrier MC, David J, Khoury ME, Lalonde L, Labelle M, Trop I. Breast Biopsies Under Magnetic Resonance Imaging Guidance: Challenges of an Essential but Imperfect Technique. Curr Probl Diagn Radiol. 2016; 45: 193-204.

17. Vreeland TJ, Berry Iv JS, Schneble E, Jackson DO, Herbert GS, Hale DF, et al. Routine Pre-Treatment MRI for Breast Cancer in a Single-Payer Medical Center: Effects on Surgical Choices, Timing and Outcomes. J Cancer. 2017; 8: $2442-8$

18. McGrath AL, Price ER, Eby PR, Rahbar H. MRI-guided breast interventions. J Magn Reson Imaging. 2017; 46: 631-45.

19. Lee CH, Dershaw DD, Kopans D, Evans P, Monsees B, Monticciolo D, et al. Breast cancer screening with imaging: recommendations from the Society of Breast Imaging and the ACR on the use of mammography, breast MRI, breast ultrasound, and other technologies for the detection of clinically occult breast cancer. J Am Coll Radiol. 2010; 7: 18-27.

20. Greenwood HI, Dodelzon K, Katzen JT. Impact of Advancing Technology on Diagnosis and Treatment of Breast Cancer. Surg Clin North Am. 2018; 98: 703-24.

21. Delille JP, Slanetz PJ, Yeh ED, Kopans DB, Garrido L. Physiologic changes in breast magnetic resonance imaging during the menstrual cycle: perfusion imaging, signal enhancement, and influence of the T1 relaxation time of breast tissue. Breast J. 2005; 11: 236-41.

22. Ellis RL. Optimal timing of breast MRI examinations for premenopausal women who do not have a normal menstrual cycle.

23. Viehweg P, Bernerth T, Heinig A, Kiechle M, Buchmann J, Koelbl H, et al. MR-guided intervention in women at high hereditary risk of breast cancer due to both family and personal history of breast cancer. Breast J. 2006; 12: 549-58.

24. Gao Y, Bagadiya NR, Jardon ML, Heller SL, Melsaether AN, Toth HB, et al. Outcomes of Preoperative MRI-Guided Needle Localization of Nonpalpable Mammographically Occult Breast Lesions. AJR Am J Roentgenol. 2016; 207: 676-84.

25. Pavic D, Koomen MA, Kuzmiak CM, Lee YH, Pisano ED. The role of magnetic resonance imaging in diagnosis and management of breast cancer. Technol Cancer Res Treat. 2004; 3: 527-41.

26. Vinnicombe S. How I report breast magnetic resonance imaging studies for breast cancer staging and screening.

27. Yeh ED, Georgian-Smith D, Raza S, Bussolari L, Pawlisz-Hoff J, Birdwell RL. Positioning in breast MR imaging to optimize image quality. Radiographics. 2014; 34: E1-17.

28. Siegler P, Holloway CM, Causer P, Thevathasan G, Plewes DB. Supine breast MRI. J Magn Reson Imaging. 2011; 34: 1212-7.

29. Carter T, Tanner C, Beechey-Newman N, Barratt D, Hawkes D. MR navigated breast surgery: method and initial clinical experience. Med Image Comput Comput Assist Interv. 2008; 11: 356-63

30. Spick C, Baltzer PA. Diagnostic utility of second-look US for breast lesions identified at MR imaging: systematic review and meta-analysis. Radiology. 2014; 273: 401-9.

31. Bumberger A, Clauser P, Kolta M, Kapetas P, Bernathova M, Helbich TH, et al. Can we predict lesion detection rates in second-look ultrasound of MRI-detected breast lesions? A systematic analysis. Eur J Radiol. 2019; 113: 96-100.

32. Spick C, Schernthaner M, Pinker K, Kapetas P, Bernathova M, Polanec SH, et al. MR-guided vacuum-assisted breast biopsy of MRI-only lesions: a single center experience. Eur Radiol. 2016; 26: 3908-16.

33. Meissnitzer M, Dershaw Dd Fau - Lee CH, Lee Ch Fau - Morris EA, Morris EA. Targeted ultrasound of the breast in women with abnormal MRI findings for whom biopsy has been recommended.

34. Meissnitzer M, Dershaw DD, Lee CH, Morris EA. Targeted ultrasound of the breast in women with abnormal MRI findings for whom biopsy has been recommended. AJR Am J Roentgenol. 2009; 193: 1025-9.

35. Lee AY, Nguyen VT, Arasu VA, Greenwood HI, Ray KM, Joe BN, et al. Sonographic-MRI Correlation After Percutaneous Sampling of Targeted Breast Ultrasound Lesions: Initial Experiences With Limited-Sequence Unenhanced MRI for Postprocedural Clip Localization. AJR Am J Roentgenol. 2018; 210: 927-34.

36. Veltman J, Boetes C, Wobbes T, Blickman JG, Barentsz JO, Magnetic resonance-guided biopsies and localizations of the breast: initial experiences using an open breast coil and compatible intervention device. Invest Radiol. 2005; 40: 379-84
37. Soo MS, Jarosz JA, Wren AA, Soo AE, Mowery YM, Johnson KS, et al. Imaging-Guided Core-Needle Breast Biopsy: Impact of Meditation and Music Interventions on Patient Anxiety, Pain, and Fatigue. J Am Coll Radiol. 2016; 13: 526-34

38. Price ER. Magnetic resonance imaging-guided biopsy of the breast: fundamentals and finer points. Magn Reson Imaging Clin N Am. 2013; 21: 571-81.

39. Mann RM, Balleyguier C, Baltzer PA, Bick U, Colin C, Cornford E, et al. Breast MRI: EUSOBI recommendations for women's information. Eur Radiol. 2015; 25: 3669-78.

40. Krug B, Hellmich M, Ulhaas A, Kramer S, Rhiem K, Zarghooni V, et al. Vacuum-assisted breast biopsies (VAB) carried out on an open 1.0T MR imager: Influence of patient and target characteristics on the procedural and clinical results. Eur J Radiol. 2016; 85: 1157-66.

41. Noroozian M, Gombos EC, Chikarmane S, Georgian-Smith D, Raza S, Denison $\mathrm{CM}$, et al. Factors that impact the duration of MRI-guided core needle biopsy. AJR Am J Roentgenol. 2010; 194: W150-7.

42. Schrading S, Simon B, Braun M, Wardelmann E, Schild HH, Kuhl CK. MRI-guided breast biopsy: influence of choice of vacuum biopsy system on the mode of biopsy of MRI-only suspicious breast lesions. AJR Am J Roentgenol. 2010; 194: 1650-7.

43. Gubern-Merida A, Marti R, Melendez J, Hauth JL, Mann RM, Karssemeijer N, et al. Automated localization of breast cancer in DCE-MRI. Med Image Anal. 2015; 20: 265-74.

44. Chang CD, Wei J, Goldsmith JD, Gebhardt MC, Wu JS. MRI guided needle localization in a patient with recurrence pleomorphic sarcoma and post-operative scarring. Skeletal Radiol. 2017; 46: 975-81.

45. Soo AE, Shelby RA, Miller LS, Balmadrid MH, Johnson KS, Wren AA, et al. Predictors of pain experienced by women during percutaneous imaging-guided breast biopsies. J Am Coll Radiol. 2014; 11: 709-16.

46. Mahoney MC, Newell MS. Breast intervention: how I do it.

47. van de Ven SM, Lin MC, Daniel BL, Sareen P, Lipson JA, Pal S, et al. Freehand MRI-guided preoperative needle localization of breast lesions after MRI-guided vacuum-assisted core needle biopsy without marker placement. J Magn Reson Imaging. 2010; 32: 101-9.

48. Zebic-Sinkovec M, Hertl K, Kadivec M, Cavlek M, Podobnik G, Snoj M. Outcome of MRI-guided vacuum-assisted breast biopsy - initial experience at Institute of Oncology Ljubljana, Slovenia. Radiol Oncol. 2012; 46: 97-105.

49. Berger N, Varga Z, Frauenfelder T, Boss A. MRI-guided breast vacuum biopsy: Localization of the lesion without contrast-agent application using diffusion-weighted imaging. Magn Reson Imaging. 2017; 38: 1-5.

50. Li J, Dershaw DD, Lee CH, Kaplan J, Morris EA. MRI follow-up after concordant, histologically benign diagnosis of breast lesions sampled by MRI-guided biopsy. AJR Am J Roentgenol. 2009; 193: 850-5.

51. Lee JM, Kaplan Jb Fau - Murray MP, Murray Mp Fau - Bartella L, Bartella L Fau - Morris EA, Morris Ea Fau - Joo S, Joo S Fau - Dershaw DD, et al. Imaging histologic discordance at MRI-guided 9-gauge vacuum-assisted breast biopsy.

52. Gombos EC, Jagadeesan J, Richman DM, Kacher DF. Magnetic Resonance Imaging-Guided Breast Interventions: Role in Biopsy Targeting and Lumpectomies. Magn Reson Imaging Clin N Am. 2015; 23: 547-61.

53. Jeffries DO, Dossett LA, Jorns JM. Localization for Breast Surgery: The Next Generation. Arch Pathol Lab Med. 2017: 141: 1324-9.

54. Kinner S, Herbrik M, Maderwald S, Umutlu L, Nassenstein K. Preoperative MR-guided wire localization for suspicious breast lesions: comparison of manual and automated software calculated targeting. Eur J Radiol. 2014; 83: e80-3.

55. Hayes MK. Update on Preoperative Breast Localization. Radiol Clin North Am. 2017; 55: 591-603.

56. Raj SD, Agrons MM, Woodtichartpreecha P, Kalambo MJ, Dogan BE, Le-Petross $\mathrm{H}$, et al. MRI-guided needle localization: Indications, tips, tricks, and review of the literature. Breast J. 2019; 25: 479-83.

57. van den Bosch MA, Daniel BL, Pal S, Nowels KW, Birdwell RL, Jeffrey SS, et al. MRI-guided needle localization of suspicious breast lesions: results of a freehand technique. Eur Radiol. 2006; 16: 1811-7.

58. Landheer ML, Veltman J, van Eekeren R, Zeillemaker AM, Boetes C, Wobbes T. MRI-guided preoperative wire localization of nonpalpable breast lesions. Clin Imaging. 2006; 30: 229-33.

59. Wang HY, Zhao YN, Wu JZ, Wang Z, Tang JH. MRI-guided wire localization open biopsy is safe and effective for suspicious cancer on breast MRI. Asian Pac J Cancer Prev. 2015; 16: 1715-8.

60. Cheang E, Ha R, Thornton CM, Mango VL. Innovations in image-guided preoperative breast lesion localization. Br J Radiol. 2018; 91: 20170740.

61. Gossmann A, Bangard C, Warm M, Schmutzler RK, Mallmann P, Lackner KJ. Real-time MR-guided wire localization of breast lesions by using an open 1.0-T imager: initial experience. Radiology. 2008; 247: 535-42.

62. Siegmann KC, Gorriz C, Xydeas T, Krainick-Strobel U, Sotlar K, Claussen CD, et al. Preoperative magnetic resonance imaging-guided localization of 131 breast lesions with modified embolization coils. Invest Radiol. 2005; 40: 368-77.

63. Johnson KS, Baker Ja Fau - Lee SS, Lee Ss Fau - Soo MS, Soo MS. Cancelation of MRI guided breast biopsies for suspicious breast lesions identified at $3.0 \mathrm{~T}$ MRI: reasons, rates, and outcomes.

64. Niell BL, Lee JM, Johansen C, Halpern EF, Rafferty EA. Patient outcomes in canceled MRI-guided breast biopsies. AJR Am J Roentgenol. 2014; 202: 223-8.

65. Plantade R, Thomassin-Naggara I. MRI vacuum-assisted breast biopsies. Diagn Interv Imaging. 2014; 95: 779-801. 
66. Boinon D, Dauchy S, Charles C, Fasse L, Cano A, Balleyguier C, et al. Patient satisfaction with a rapid diagnosis of suspicious breast lesions: Association with distress and anxiety. Breast J. 2018; 24: 154-60.

67. Mann RM, Mus RD, van Zelst J, Geppert C, Karssemeijer N, Platel B. A novel approach to contrast-enhanced breast magnetic resonance imaging for screening: high-resolution ultrafast dynamic imaging. Invest Radiol. 2014; 49: $579-85$

68. Chhor CM, Mercado CL. Abbreviated MRI Protocols: Wave of the Future for Breast Cancer Screening. AJR Am J Roentgenol. 2017; 208: 284-9.

69. Ertas G, Doran SJ, Leach MO. A computerized volumetric segmentation method applicable to multi-centre MRI data to support computer-aided breast tissue analysis, density assessment and lesion localization. Med Biol Eng Comput. 2017; 55: 57-68.

70. Neil JJ. Diffusion imaging concepts for clinicians. J Magn Reson Imaging. 2008; 27: 1-7.

71. Partridge SC, Demartini WB, Kurland BF, Eby PR, White SW, Lehman CD. Differential diagnosis of mammographically and clinically occult breast lesions on diffusion-weighted MRI. J Magn Reson Imaging. 2010; 31: 562-70.

72. Partridge SC, McDonald ES. Diffusion weighted magnetic resonance imaging of the breast: protocol optimization, interpretation, and clinical applications. Magn Reson Imaging Clin N Am. 2013; 21: 601-24.

73. Park SB, Kim JG, Lim KW, Yoon CH, Kim DJ, Kang HS, et al. A magnetic resonance image-guided breast needle intervention robot system: overview and design considerations. Int J Comput Assist Radiol Surg. 2017; 12: 1319-31.

74. Barth RJ, Jr., Krishnaswamy V, Paulsen KD, Rooney TB, Wells WA, Rizzo E, et al. A Patient-Specific 3D-Printed Form Accurately Transfers Supine MRI-Derived Tumor Localization Information to Guide Breast-Conserving Surgery. Ann Surg Oncol. 2017; 24: 2950-6.

75. Green M, Vidya R. Techniques Used to Localize Occult Breast Lesions: An Update. Clin Breast Cancer. 2018; 18: e281-e3.

76. Philadelpho Arantes Pereira F, Martins G, Gregorio Calas MJ, Fonseca Torres de Oliveira MV, Gasparetto EL, Barbosa da Fonseca LM. Magnetic resonance imaging-radioguided occult lesion localization (ROLL) in breast cancer using Tc-99m macro-aggregated albumin and distilled water control. BMC Med Imaging. 2013; 13: 33.

77. de Lima Docema MF, Costa PA, de Andrade FE, Bevilacqua JL, Elias S, Cerri GG, et al. Magnetic resonance imaging-guided occult breast lesion localization and simultaneous sentinel lymph node mapping. World J Surg Oncol. 2014; 12: 320 .

78. Ruiz-Delgado ML, Lopez-Ruiz JA, Saiz-Lopez A. Abnormal mammography and sonography associated with foreign-body giant-cell reaction after stereotactic vacuum-assisted breast biopsy with carbon marking. Acta Radiol. 2008; 49: 1112-8

79. Al-Hilli Z, Glazebrook KN, McLaughlin SA, Chan DM, Robinson KT, Giesbrandt JG, et al. Utilization of Multiple I-125 Radioactive Seeds in the Same Breast is Safe and Feasible: A Multi-institutional Experience. Ann Surg Oncol. 2015; 22: 3350-5.

80. Cox CE, Garcia-Henriquez N, Glancy MJ, Whitworth P, Cox JM, Themar-Geck M, et al. Pilot Study of a New Nonradioactive Surgical Guidance Technology for Locating Nonpalpable Breast Lesions. Ann Surg Oncol. 2016; 23: 1824-30.

81. Price ER, Khoury AL, Esserman LJ, Joe BN, Alvarado MD. Initial Clinical Experience With an Inducible Magnetic Seed System for Preoperative Breast Lesion Localization. AJR Am J Roentgenol. 2018; 210: 913-7.

82. Imschweiler T, Haueisen H, Kampmann G, Rageth L, Seifert B, Rageth C, et al. MRI-guided vacuum-assisted breast biopsy: comparison with stereotactically guided and ultrasound-guided techniques. Eur Radiol. 2014; 24: 128-35.

83. Bick U, Trimboli RM, Athanasiou A, Balleyguier C, Baltzer PAT, Bernathova $\mathrm{M}$, et al. Image-guided breast biopsy and localisation: recommendations for information to women and referring physicians by the European Society of Breast Imaging. Insights Imaging. 2020; 11: 12.

84. Goto M, Ito H, Akazawa K, Kubota T, Kizu O, Yamada K, et al. Diagnosis of breast tumors by contrast-enhanced MR imaging: comparison between the diagnostic performance of dynamic enhancement patterns and morphologic features. J Magn Reson Imaging. 2007; 25: 104-12

85. DeMartini W, Lehman C, Partridge S. Breast MRI for cancer detection and characterization: a review of evidence-based clinical applications. Acad Radiol. 2008; 15: 408-16.

86. Wallis M, Tardivon A, Helbich T, Schreer I, European Society of Breast I. Guidelines from the European Society of Breast Imaging for diagnostic interventional breast procedures. Eur Radiol. 2007; 17: 581-8.

87. Orel SG, Rosen M, Mies C, Schnall MD. MR imaging-guided 9-gauge vacuum-assisted core-needle breast biopsy: initial experience. Radiology. 2006; 238: 54-61.

88. Lehman CD, Deperi ER, Peacock S, McDonough MD, Demartini WB, Shook J. Clinical experience with MRI-guided vacuum-assisted breast biopsy. AJR Am J Roentgenol. 2005; 184: 1782-7.

89. Hauth EA, Jaeger HJ, Lubnau J, Maderwald S, Otterbach F, Kimmig R, et al. MR-guided vacuum-assisted breast biopsy with a handheld biopsy system: clinical experience and results in postinterventional MR mammography after 24 h. Eur Radiol. 2008; 18: 168-76.

90. Taneja S, Jena A, Kumar K, Mehta A. Technical Note: MRI-guided breast biopsy - our preliminary experience. Indian J Radiol Imaging. 2010; 20: 218-20.

91. Brenner RJ, Gordon LM. Malignant seeding following percutaneous breast biopsy: documentation with comprehensive imaging and clinical implications. Breast J. 2011; 17: 651-6.
92. Santiago L, Adrada BE, Huang ML, Wei W, Candelaria RP. Breast cancer neoplastic seeding in the setting of image-guided needle biopsies of the breast. Breast Cancer Res Treat. 2017; 166: 29-39.

93. Meeuwis C, Peters Nh Fau - Mali WPTM, Mali Wp Fau - Gallardo AMF, Gallardo Am Fau - van Hillegersberg R, van Hillegersberg R Fau - Schipper MEI, Schipper Me Fau - van den Bosch MAAJ, et al. Targeting difficult accessible breast lesions: MRI-guided needle localization using a freehand technique in a $3.0 \mathrm{~T}$ closed bore magnet.

94. CD L, ER D, S P, MD M, WB D, roentgenology SJJAAjo. Clinical experience with MRI-guided vacuum-assisted breast biopsy. 2005; 184: 1782-7.

95. Malhaire C, El Khoury C, Thibault F, Athanasiou A, Petrow P, Ollivier L, et al. Vacuum-assisted biopsies under MR guidance: results of 72 procedures. Eur Radiol. 2010; 20: 1554-62.

96. An Y, Kim S, Kang B, Lee J. Usefulness of magnetic resonance imaging-guided vacuum-assisted breast biopsy in Korean women: a pilot study. World J Surg Oncol. 2013; 11: 200.

97. Causer PA, Piron CA, Jong RA, Curpen BN, Luginbuhl CA, Glazier JE, et al. MR imaging-guided breast localization system with medial or lateral access. Radiology. 2006; 240: 369-79.

98. Myers KS, Kamel IR, Macura KJ. MRI-guided breast biopsy: outcomes and effect on patient management. Clin Breast Cancer. 2015; 15: 143-52.

99. Taskin F, Soyder A, Tanyeri A, Ozturk VS, Unsal A. Lesion characteristics, histopathologic results, and follow-up of breast lesions after MRI-guided biopsy. Diagn Interv Radiol. 2017; 23: 333-8.

100. Wang J, Song Y, Liu J, Meng X, Xing Z, Zhang M, et al. Clinical Application and Feasibility of MRI-Guided Breast Biopsy of Breast Minimal Lesions in Chinese Population. Front Oncol. 2020; 10: 257

101. Ferre R, Ianculescu V, Ciolovan L, Mathieu MC, Uzan C, Canale S, et al. Diagnostic Performance of MR-guided Vacuum-Assisted Breast Biopsy: 8 Years of Experience. Breast J. 2016; 22: 83-9. 